Original Research Paper

\title{
Straight Line Routing on Next Hop Graph in Wireless Sensor Networks
}

\author{
Kavitha Rani, P. and E. Kannan \\ Department of CSE, Vel Tech University, Chennai, India
}

\author{
Article history \\ Received: $15-06-2014$ \\ Revised: 06-08-2014 \\ Accepted: 09-08-2014 \\ Corresponding Author: \\ Kavitha Rani \\ Department of CSE, \\ Vel Tech University, \\ Chennai, India \\ Email: kavits.05@gmail.com
}

\begin{abstract}
Information processing and information routing in wireless sensor network is one of the most important key area of recent research. Building an optimal routing protocol for information routing is a tough task in dealing with the sensor nodes. Information exchange can be done with the major possible way in order to provide a secure communication and reliable data delivery, here in this study we focus on routing protocol with reliable delivery. Hence we proposed a new algorithm/protocol which optimally routes the data packets from source to destination with efficient utilization of energy resources. The proposed protocol is based on next hop graph node in terms of reliable data packet routing by framing the route in the form of graphs. The simulation results showed in the performance analysis phase denotes the proposed method outperforms the existing routing protocol in terms of reliability and robustness in efficient routing. The proposed methodology achieves the connectivity and connectivity path in WSN which is critical in WSN.
\end{abstract}

Keywords: WSN, Straight Line Routing, Next Hop Graph, Routing

\section{Introduction}

With major advances in technology, sensors have become more power with a smaller in size at a low cost that has improved the effectiveness of many military and social applications such as battle field surveillance, protection and tragedy management. (Baghyalakshmi et al., 2010; Barabási and Albert, 1999; Broch et al., 1999) Wireless Sensor network is an advanced wireless sensor technology used in various applications such as medical-health monitoring, military applications (Guo and Jia, 2012; Karp and Kung, 2000; Kennedy and Eberhart. 1995). One of the most vital problems in wireless sensor networks is finding optimal routes for transmitting data between sources to destination. (Ruyan et al., 2010; Satyanarayanan, 2007) Generally it pairs in a multi-hop fashion. Several algorithms have been proposed for routing. The previous research for routing scheme was explained clearly in the section "previous work". Arif and Rani, (2012; Okdem and Karaboga, 2009) Many researchers have undergone for solving the routing problem in Wireless sensor network.

WSN consist of large numbers (hundreds or thousands) of wirelessly connected heterogeneous sensor nodes that are spatially scattered across a large field of interest (Kavitha Rani and Kannan, 2013) and the process continuous with data gathering from several sensors to monitor events. (Park and Corson, 1997; Rao et al., 2003) In this study, we proposed a robust methodology to exchange data packets with less energy consumption and with high processing of data exchange, a new hybrid algorithm has been developed and built for data routing.

\section{Previous Work}

Various researchers have proposed various optimal routing algorithms for information routing in Wireless sensor networks. Since the need of finding optimality has not reached the end source, the expectation for the routing protocol from its source is still in need. Previous attempt was finding optimal path using hybrid 
scheme which combines PSO algorithms with the computational intelligence. In that way, the initial stage of research started with FFA algorithm which is basically fast reactive and fast adaptive in nature. The protocol is insisted by the SI and advanced computational procedure to route the data packets. The main key idea behind the protocol is to navigate the packets even if the node failure occurs. The node head and node defined are clearly explained in the section Local field where the packet information and routing information are stored. The clear cut explanation of the node selection and inspired swarm based formulation (Kavitha Rani and Kannan, 2013):

$$
V_{i}^{t+1}=x\left[V_{i}^{t}+\sum_{\rho k \epsilon \aleph_{i}} \varphi_{k} U_{k}^{t}\left(p b_{k}^{t}+x_{k}^{t}\right)\right]
$$

where, $V_{i}^{t}$ denotes the velocity grid of the node.

For Node-Head selection the end solution formulated was defined as where:

$$
V_{i}^{t}=A v^{t}+b 1 U_{1}^{t}\left(p b^{t}-x^{t}\right)+b 2 U_{2}^{t}\left(g b^{t}-x^{t}\right)
$$

\section{Proposed Methodology}

Here a new hybrid methodology is used with swarm intelligence and basic approach in computational efficiency. The proposed methodology has high efficiency in terms of utilizing the energy resources. Visu et al. (2012; Yong-Chang and Gang, 2008) Various approaches are worked with in order to achieve high optimality and to route the data packets. The main key idea is to provide node identity with packet information which was achieved in the previous attempt in the field of Local field information. Next hop of each sensor nodes are traced back using the FFA algorithm, once the node has been identified the hop count will raises to next level, the graph predicts the node value of each vertices to be in the straight line, thus the graph predicted forms a minimal rate of spanning tree using the vertices. The hop count will be in increasing order which helps in identifying the node and its counter.

The Next hop is predicted by flooding protocol which floods the ARP req i.e., RTS message to all the neighbours, once the node which is nearest to the flooding node, the particular node responds to the corresponding node with CTS message. Once the CTS message is received the node is identified and its distance vector is initialized in the Hop counter. Thus the counter is incremented whenever it receives CTS message from various neighbours.

\section{Next Hop Graph}

Next hop graph is generated using the neighbour node ACK packets. When it receives the more number of packets the counter will be incremented based on the response from various nodes. The zone level is indicated based on localization of the sensor nodes. Once the node is identified then the vertices of each node are updated and plotted into the graph structure. The node with higher range of localized positioning takes the lead of all the nodes and acts as the root/ head of all the nodes.

\section{Zone Level}

Zone is defined based on the localization distance of the sensor node. Sedighizadeh and Masehian (2009; Shen et al., 2009) The distance is calculated by means of acquiring the response time with respect to the distance of the nearest node which is in active state:

$$
\text { Distance }=\text { Localization value }-\frac{\text { Response time of the sensor }}{\text { distance of thenearest neighbour }}
$$

\section{Algorithm}

Function straightlinerouting(Input, output)

Input: Number of nodes

Output: Node localization, data packet routing

Initialize (all);

Init.WSN( start,dest, pkt_size,protocol_stack);

Start_node $=1$;

Dest_node $=$;

Init.run();

Start.setMode(flood());

Receiver( MAC, Bind_addr, SYN);

Counter++;node+1;

If Counter.value $<$ numberof nodes

Traverse all the vertices

Else

$$
\text { V1 = Plot.vertices }() \text {; }
$$$$
\text { Graph(v1); }
$$

Display(graph());

End if

Print route_img;

End

\section{Result Analysis}

The proposed methodology is tested with maximum of 30 sensor nodes and minimum of 5 sensor nodes. 


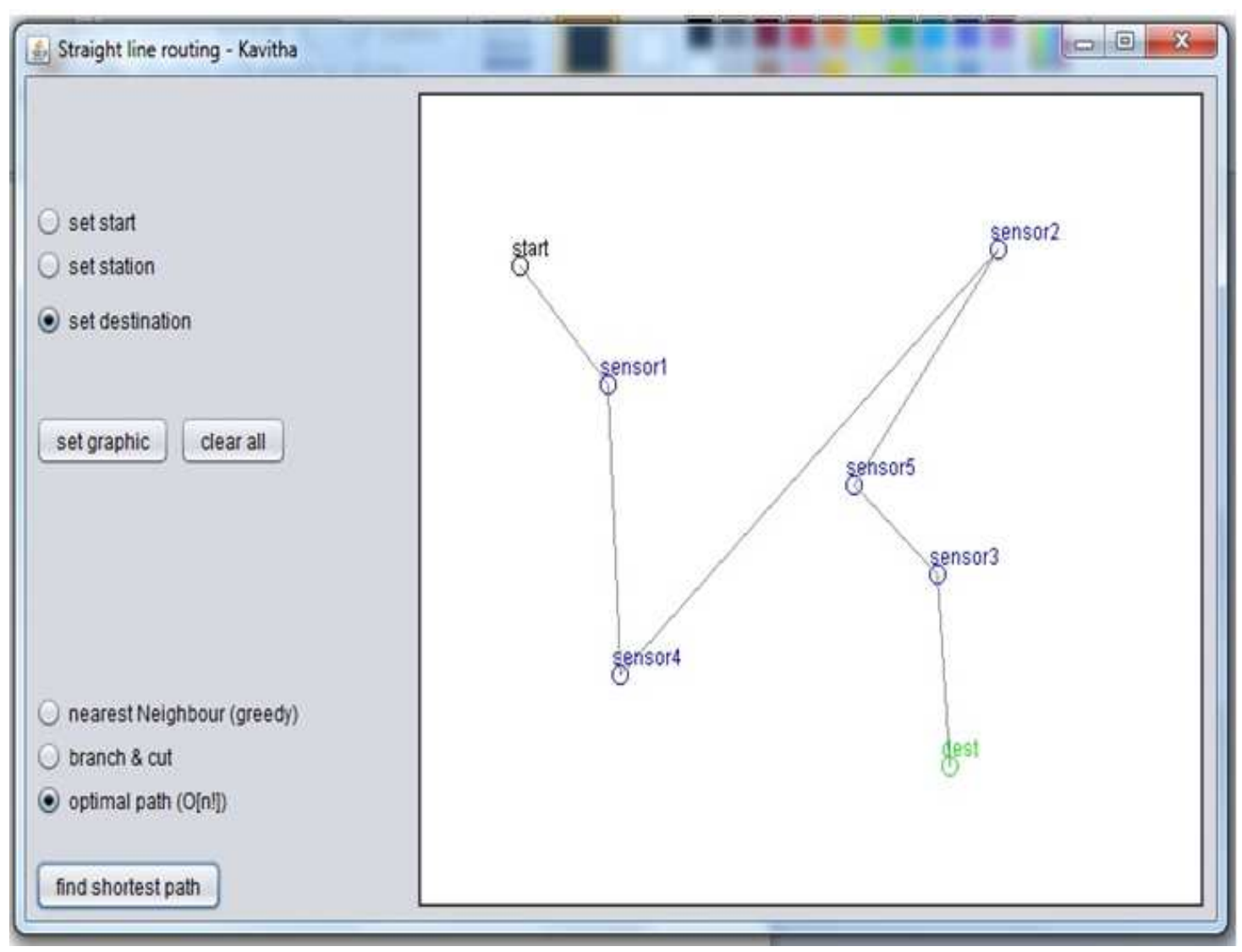

Fig. 1. Optimal path strategy

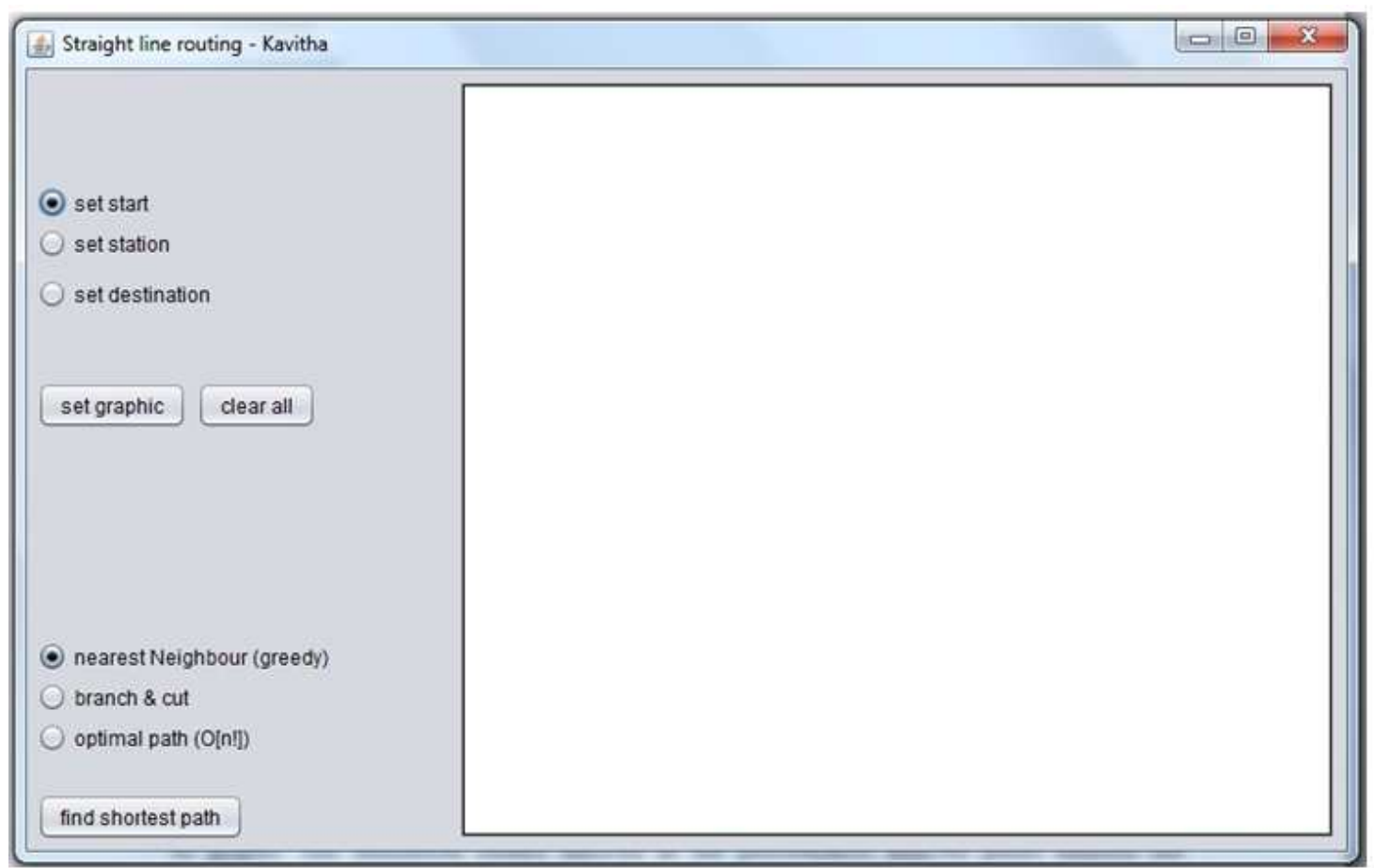

Fig. 2. Simulating working model of the proposed protocol 


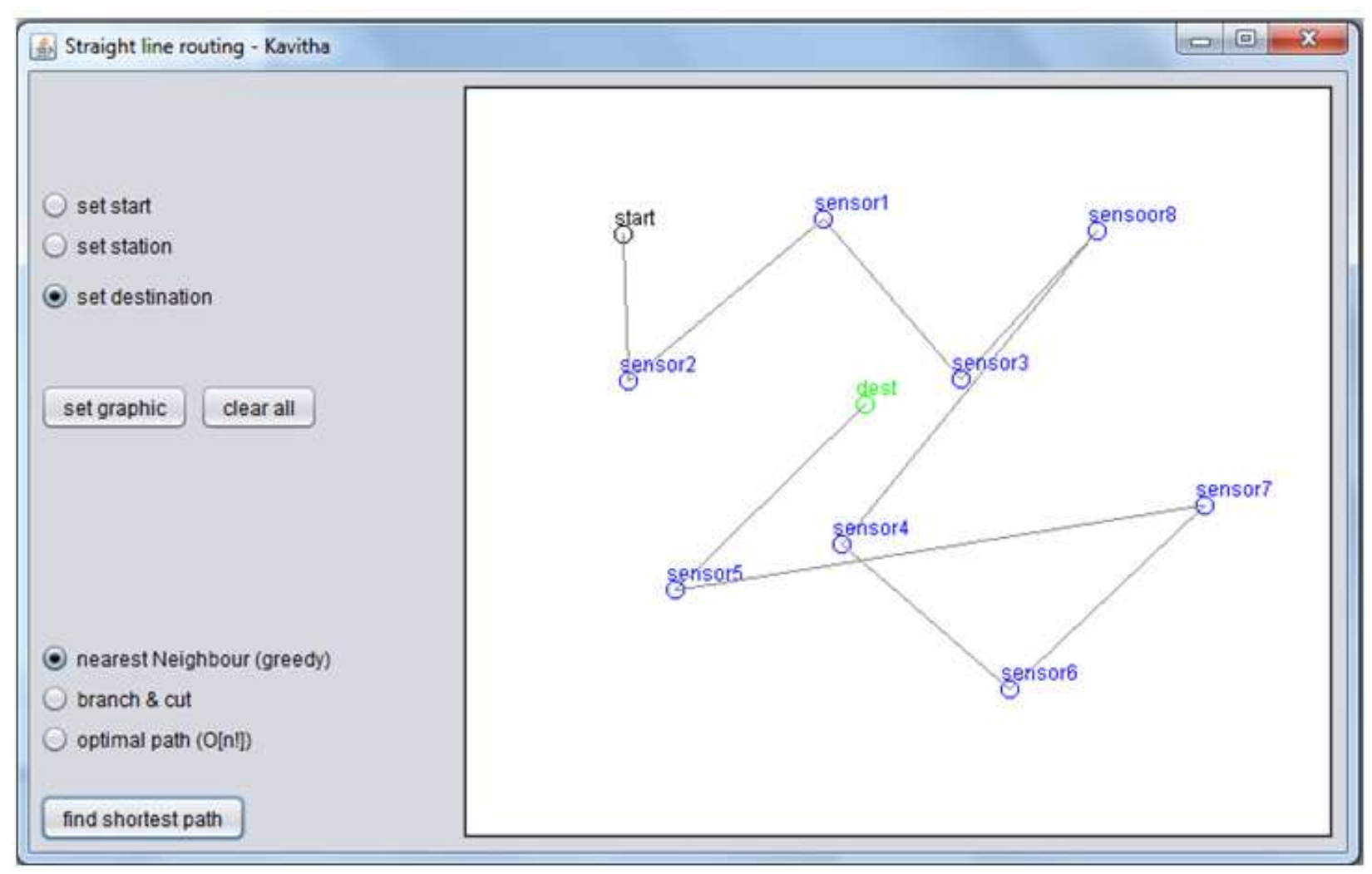

Fig. 3. Nearest neighbour strategy

The simulation model of the proposed protocol has been implemented in JAVA platform and an efficient simulator of the proposed protocol has been built for WSN and Adhoc networks. The implementation results are clearly defined in the figure which states the actual working principle of the proposed protocol and straight line routing in technical aspects. Figure denotes the environment of the sensor nodes to be place. Figure shows the nearest neighbour finding in the proposed methodology. Figure clearly defines the proposed routing model with optimal path found by using various schemes which was stated in the section "next hop graph".

Figure clearly denotes the working model of straight line routing.

Figure which states the actual working principle of the straight line routing with active nearest neighbour vertices.

\section{Conclusion}

Here we conclude the paper with achieving better results in hybrid routing schemes, the proposed methodology outperforms with $91 \%$ accuracy in terms of data packet routing and the scheme supports the security orientation during the data transmission due to its CTS and RTS flooding mechanism. It combines various localization features such as flooding, root node prediction, nearest neighbour discovery. Here the methodology is well defined and it can be used for various applications such as military intrusion detection system, medical applications. Figure 1 shows that the proposed methodology can be a root of adaptation to various higher level application tier protocols and hence it can be easily adapted to Mobile Adhoc based networks. Figure 2 and 3 shows the simulator working model of the proposed protocol. Hence the enhancement level of the proposed protocol will be on Adhoc networks-information routing and its strategy on reliable packet delivery. Development of next strategic level routing will be on branch and cut methodology towards the future scope of development on MANET and WSN (Shirkande and Vatti, 2013; Sombuntham and Kachitvichayanukul, 2010).

\section{Acknowledgment}

Hence I thank Dr. E. Kannan and Dr. P. Visu for their strong support in carrying my research 


\section{Author's Contributions}

All authors equally contributed in this study.

\section{Ethics}

This article is original and contains unpublished material. The corresponding author confirms that all of the other authors have read and approved the manuscript and no ethical issues involved.

\section{References}

Baghyalakshmi, D., J. Ebenezer and S.A.V. Satyamurty, 2010. Low latency and energy efficient routing protocols for wireless sensor networks. Proceedings of the International Conference on Wireless Communication and Sensor Computing, Jan. 2-4, IEEE Xplore Press, Chennai, pp: 1-6.

DOI: 10.1109/ICWCSC.2010.5415892

Barabási, A.L. and R. Albert, 1999. Emergence of scaling in random networks. Science, 286: 509-512. DOI: 10.1126/science.286.5439.509

Broch, J., D.B. Johnson and D.A. Maltz, 1999. The dynamic source routing protocol for mobile ad hoc networks. Internet-Draft Version.

Guo, D. and C. Jia, 2012. A WSN routing algorithm using overpayment on complex network. Proceedings of the 5th International Conference on Intelligent Computation Technology and Automation, Jan. 12-14, IEEE Xplore Press, Zhangjiajie, Hunan, pp: 53-56.

DOI: 10.1109/ICICTA.2012.20

Karp, B. and H.T. Kung, 2000. Gpsr: Greedy perimeter stateless routing for wireless networks. Proceedings of the 6th Annual International Conference on Mobile Computing and Networking, Aug. 06-11, ACM New York, pp: 243-254. DOI: $10.1145 / 345910.345953$

Kavitha Rani, P. and E. Kannan, 2013. FFA-context aware energy efficient routing using fast reactive and adaptive algorithm. Am. J. Applied Sci., 11: 301-307. DOI: 10.3844/ajassp.2014.301.307

Kennedy, J. and R. Eberhart, 1995. Particle swarm optimization. Proceedings of the IEEE International Conference on Neural Networks, Nov. 27-Dec 01, IEEE Xplore Press, Perth, WA., pp: 1942-1948.

DOI: $10.1109 /$ ICNN.1995.488968

Arif, M. and T. Rani, 2012. ACO based routing for MANETS. Int. J. Wireless Mobile Netw., 4: 163-174.
Okdem, S. and D. Karaboga, 2009. Routing in wireless sensor networks using an Ant Colony Optimization (ACO) router chip. Sensors, 9: 909-921. DOI: 10.3390/s90200909

Park, D.V. and M.S. Corson, 1997. A highly adaptive distributed routing algorithm for mobile wireless networks. Proceedings of the IEEE 16th Annual Joint Conference of the IEEE Computer and Communications Societies Driving the Information Revolution, Apr. 7-12, IEEE Xplore Press, Kobe, pp: 1405-1413.

DOI: 10.1109/INFCOM.1997.631180

Rao, A., S. Ratnasamy, C. Papadimitriou, S. Shenker and I. Stoica, 2003. Geographic routing without location information. Proceedings of the 9th Annual International Conference on Mobile Computing and Networking, Sept. 14-19, ACM New York, pp: 96-108. DOI: $10.1145 / 938985.938996$

Ruyan, Z., C. Ming, F. Guofu, L. Huifang and H. Shijun, 2010. Genetic clustering route algorithm in WSN. Proceedings of the 6th International Conference on Natural Computation, Aug. 10-12, IEEE Xplore Press, Yantai, Shandong, pp: 4023-4026.

DOI: $10.1109 /$ ICNC.2010.5584826

Satyanarayanan, D., 2007. Greedy local delaunay triangulation routing for wireless ad hoc networks. Proceedings of the International Conference on Signal Processing, Communications and Networking, Feb. 22-24, Chennai, pp: 49-53. DOI: 10.1109/ICSCN.2007.350694

Sedighizadeh, D. and E. Masehian, 2009. Particle swarm optimization methods, taxonomy and application. Int. J. Comput. Theory Eng., 1: 486-502. DOI: 10.7763/IJCTE.2009.V1.80

Shen, H., Y. Zhu, T. Liu and L. Jin, 2009. Particle swarm optimization in solving vehicle routing problem. Proceedings of the 2nd International Conference on Intelligent Computation Technology and Automation, Oct. 10-11, IEEE Xplore Press, Changsha, Hunan, pp: 287-291. DOI: 10.1109/ICICTA.2009.77

Shirkande, S.D. and R.A. Vatti, 2013. ACO based routing algorithms for ad-hoc network (WSN, MANETs): A survey. Proceedings of the International Conference on Communication Systems and Network Technologies, Apr. 6-8, IEEE Xplore Press, Gwalior, pp: 230-235.

DOI: $10.1109 /$ CSNT.2013.56 
Sombuntham, P. and V. Kachitvichayanukul, 2010. A Particle swarm optimization algorithm for multidepot vehicle routing problem with pickup and delivery requests.

Visu, P., S. Koteeswaran and J. Janet, 2012. Artificial bee colony based energy aware and energy efficient routing protocol. J. Computer Sci., 8: 227-231. DOI: $10.3844 /$ jcssp.2012.227.231
Yong-Chang, Y. and W. Gang, 2008. Load balance data gathering algorithm in wireless sensor networks. J. Commun. Technol., 41: 92-95. 